\title{
Pensamiento táctico y efectividad en lanzadores de béisbol
}

Tactical thinking and effectiveness in baseball pitchers

\section{Hechavarría Pérez, Angel Manuel ${ }^{1}$ y Candia Luján, Ramón ${ }^{1}$}

${ }^{1}$ Universidad Autónoma de Chihuahua, México

Miguel de Cervantes 209-47, Paseos de Chihuahua, Chihuahua, México

\section{CORRESPONDENCIA}

\section{Angel Manuel Hechavarría Pérez:}

ahechavarriaperez@gmail.com

Universidad Autónoma de Chihuahua

\section{CÓMO CITAR}

Hechavarría, A. M. y Candia, R. (2020). Pensamiento táctico y efectividad en lanzadores $\mathrm{d}$ béisbol. Revista de Ciencias del Ejercicio, 15 (1), pp. 48-58. Recuperado de: revistafod.uanl.mx

\section{RESUMEN}

El presente estudio tuvo como objetivo fundamental determinar la relación que existe entre pensamiento táctico y efectividad de los lanzadores del equipo, Dorados de chihuahua que participó en al campeonato estatal del 2019. Para ello se utilizó un cuestionario o test que consta de 40 preguntas de reflexión y análisis referidas a la táctica y estrategia que debe llevar a cabo, el lanzador en su enfrentamiento al bateador, es un estudio no experimental, transversal, la muestra estuvo integrada por 14 lanzadores que formaron parte del staff a los cuales se les aplico el cuestionario y luego se comparó con la efectividad que tuvieron en cuanto a promedio de carreras limpias durante el campeonato. Como principales resultados se pudo conocer que los lanzadores que mejores resultados obtuvieron en el test coinciden en más del $80 \%$ con los de mejores resultados en cuanto a promedio de carreras limpias. Las conclusiones arrojaron que existe una relación directa entre el pensamiento táctico y los resultados de los lanzadores del equipo Dorados de Chihuahua.

Palabras clave: táctica, pensamiento táctico, béisbol.

\section{ABSTRACT}

The main objective of the present study was to determine the relationship between tactical thinking and the effectiveness of the team's pitchers, Dorados de Chihuahua, who participated in the 2019 state championship. To do this, a questionnaire or test consisting of 40 reflection questions was used. and analysis referring to the tactic and strategy to be carried out, the pitcher in his confrontation with the batter, is a non-experimental, cross-sectional study, the sample consisted of 14 pitchers who were part of the staff to which the questionnaire was applied and then it was compared to the effectiveness they had in terms of average clean runs during the championship. As main results, it was known that the pitchers who obtained the best results in the test coincide in more than $80 \%$ with those with the best results in terms of average clean runs. The conclusions showed that there is a direct relationship between tactical thinking and the results of the launchers of the Dorados de Chihuahua team.

Key words: tactic, tactical thinking, baseball. 
Materiales y métodos

Para muchos entendidos y estudiosos de la rama del deporte, el béisbol es llamado por derecho propio el rey de los deportes, ya que en este tan maravilloso juego se conjugan muchas habilidades, destrezas, capacidades físicas, cualidades volitivas y habilidades mentales que son indispensables para obtener altos logros deportivos.

Dentro del béisbol, el pitcheo constituye la principal arma a la hora de encarar un juego, una competencia o un torneo a corta, media o larga duración, se plantea que en más del $70 \%$ de la victoria en un juego depende del trabajo que sea capaz de realizar el lanzador.

En los últimos años tanto entrenadores como buscadores de talentos le han atribuido mayor importancia a las características y cualidades físicas de los jugadores que a la inteligencia y operatividad emocional que poseen los jóvenes, a la hora de seleccionarlos, en ese sentido solo se aplican pruebas físicas de velocidad, potencia de brazo y swing y no se realizan test mentales que midan las cualidades volitivas así como inteligencia emocional e intelectual, lo que trae consigo que dichos jugadores sobre todo en el área de lanzadores cuando tengan que enfrentarse a situaciones difíciles en su duelo con los bateadores salgan con resultados desfavorables.

Dentro de un juego de béisbol la principal lucha táctica se centra entre el lanzador y el bateador, teniendo teóricamente mayor ventaja el lanzador pues el sí sabe lo que va a lanzar, pero no así el bateador que debe adivinar qué tipo de lanzamiento le rea- lizarán, así como la zona hacia donde será dirigido el mismo.

La táctica es el proceso en que se conjugan todas las posibilidades físicas, técnicas, teóricas, psicológicas y demás, para dar una solución inmediata a las disímiles situaciones imprevistas y cambiantes que se crean en condiciones de oposición (Álvarez, 2002).

El pensamiento táctico es determinante en los deportes donde la estrategia y la táctica juegan un papel fundamental, el mismo forma parte de los componentes de la preparación, incluyéndose en la preparación táctica, teórica y psicológica, por lo que se le debe dar una importancia capital durante el proceso de entrenamiento sobre todo en los deportes de oposición.

Debido al alto nivel de enfrentamiento y grandes exigencias competitivas actualmente poseer un alto nivel racional y pensamiento táctico creador ha cobrado mayor importancia lo cual es expresado en la selección por parte del jugador, de la estrategia y táctica más adecuada para contrarrestar la acción del contario fundamentalmente en el enfrentamiento lanzador vs bateador.

La táctica o pensamiento táctico se divide en tres fases fundamentales percepción, análisis y ejecución. En ese sentido el lanzador antes de ejecutar un lanzamiento primeramente debe percibir la información (las señas de su receptor, colocación del bateador, tipo de bateador, situación del juego...), debe analizar para darle solución mental a la acción y por último ejecutar la acción motriz (Mhalo,1974). 
Rosales, (2016) plantea que la táctica es la forma más eficiente de realizar la técnica, empleando el menor gasto energético posible.

Autores tales como Freidich Mahlo (1974); Matveev, L., (1983), Harre, D., (1983), Talaga J., (1989); Gómez, P. E. y Peñaloza, R., (2001); Negreira, J., (2002); Castejón Oliva. F, J (2005) centran sus ideas en que la táctica se expresa a través de un pensamiento productivo que permite conducir la lucha deportiva, la reconocen como teoría de la conducción del encuentro deportivo; como forma examinada y juiciosa de la competencia deportiva con el contrario regida por un reglamento y basado en las capacidades y la colaboración de los distintos jugadores que se adaptan a las condiciones, cuyo objetivo es alcanzar altos logros deportivos.

Las tácticas puestas en juego en el pasado por los directores de prestigio son estudiadas para modificarlas, de modo que resulten útiles bajo las condiciones actuales. Por lo que en cada lanzamiento utilizado por el lanzador en un juego de béisbol está implícita una acción táctica. La capacidad de observación, análisis, la memoria, el pensamiento, la concentración y las sensaciones, son importantes y deben actuar entrelazados e influenciados mutuamente para lograr una buena acción (Cartaya, 2009).

El lanzador y su entrenador deben ser muy estudiosos y tener en cuenta varios factores que se ponen de manifiesto en cada acción del juego, lanzamiento por lanzamiento ya que una equivocación puede constituir sencillamente la pérdida del partido.
Un lanzador de nivel deberá entrenar su capacidad para resolver los problemas que genera el juego seleccionando información precisa. Del reporte de datos (score, outs, innings, cuenta del bateador, corredores en base, jugadores involucrados y el uso de "patrones de generalización") que le servirán para establecer su plan táctico de pitcheo.

Por lo anteriormente expuesto se puede resaltar que tanto la inteligencia y operatividad emocional son cualidades que pueden permitir que un jugador tenga un pensamiento táctico adecuado que se pongan de manifiesto a las circunstancias de juego.

El objetivo del presente estudio fue determinar la relación que existe entre el pensamiento táctico y la efectividad de los lanzadores del equipo Dorados de Chihuahua, en el torneo estatal 2019.

\section{Materiales y métodos}

Para la investigación se tomó como base el protocolo sobre pensamiento táctico diseñado por J, R, Alvaredo (1993) citados por A. Pedreira, (1993, 1994, 1995, Pérez Pardo y Pérez Zurita 2003) y Cartaya 2009 el cual da un diagnóstico del pensamiento táctico y su correlación con los resultados y efectividad de uno o varios lanzadores.

El mismo consta de 50 preguntas para responder con 4 posibles respuestas pre elaboradas, aunque en este caso solo se escogieron 40 preguntas teniendo en cuenta el nivel intelectual de los participantes y las diferencias existentes entre los participantes en cuanto a edad, tiempo en el deporte, temporadas en el equipo etc. 
Diseño: trasversal, no experimental de muestreo no probabilístico intencionado, para ello se le pidió el consentimiento a la dirección del equipo, así como a los directivos de dicha institución deportiva los cuales proporcionaron los recursos humanos, así como los medios y materiales mínimos indispensables.

Muestra: La selección de la muestra estuvo conformada por 14 lanzadores que conformaron el equipo Dorados de Chihuahua que participó en el campeonato estadal del estado del mismo nombre, las edades de los sujetos están comprendidas de entre 17 y 32 años de edad con un promedio de tiempo en el deporte de 12 años como media.

Criterios de inclusion:

- Que los encuestados no presenten ningún trastorno o afección de tipo psicológico.

- Lanzadores que al final del campeonato hayan tenido como mínimo 7 entradas lanzadas.

- El consentimiento y disposición de los jugadores, dirección y directivos del equipo para formar parte de nuestra investigación.

- La selección de la muestra previamente evaluada por el psicólogo del equipo.

\section{Instrumento:}

Se aplicó el test de pensamiento táctico elaborado por el doctor Juan Ramón Alvaredo, Psicólogo del equipo nacional de Béisbol de Cuba con más de 30 años de experiencia en dichas funciones, dicho protocolo está homologado por el comité de ética del instituto superior Manuel fajardo de la ciudad de la $\mathrm{Ha}$ bana, Cuba. El cuestionario consta de 40 preguntas, todas relacionadas con acciones de juego basadas fundamentalmente al enfrentamiento entre lanzador y bateador. Cada pregunta tiene 4 posibles respuestas una de ella es positiva, una es aceptable y una negativa, además se incluye una extra que siempre será No sé, que el sujeto elegirá cuando no es capaz de analizar coherentemente el contenido de la misma.

A partir de los resultados obtenidos del cuestionario de 40 preguntas que establece la tabla donde cada respuesta positiva tiene un valor de 1 punto, negativa 0 pero existe una bonificación de 0.5 puntos a aquellas posibles respuestas que pudieran constituir una segunda opción tomada de acuerdo a las características de cada lanzador, justificando el porqué de su respuesta.

Procedimiento: Para llevar a cabo la aplicación del test primero se sentó a cada sujeto en una mesa y se le entregó el cuestionario donde debían plasmar su nombre y apellidos e ir respondiendo con la mayor sinceridad y honestidad posible cada una de las preguntas, las cuales tenían cuatro posibles respuestas, debiendo marcar solamente una con una hora de duración, una vez terminado se recopilan para su análisis individual y general.

Análisis de datos:

Para el análisis de los datos se toma como referencia las respuestas pre elaboradas y respondidas, aprobadas por expertos de la dirección nacional de béisbol de cuba y homologada por la federación internacional de béisbol para su generalización. 
El análisis estadístico se realizó tomando los datos obtenidos en el test, así como las estadísticas oficiales proporcionadas por el sitio web de la asociación estatal de béisbol del estado Chihuahua. Empleándose para ello tablas de contingencia que permitieron localizar los detalles más significativos mediante el empleo del paquete SPSS, versión 25 con un $p$ valor $>0.05$, donde se realizó la sumatoria de los puntos obtenidos en cada una de las preguntas (40), la media general, así como la evaluación puntual de cada una de las preguntas y se hace un análisis de correlación entre las variables evaluativas de promedio de carreras limpias por juegos y puntuación obtenida en el test.

Para la normalidad de los datos se realizó la prueba Shapiro wilk y la variable promedio de carreras limpias no estaba normalmente distribuidas por lo que se pasa a realizar la prueba no paramétrica de chi cuadrado obteniéndose los siguientes resultados. Tabla 4, anexos.

Para la evaluación de los lanzadores en cuanto a puntos se toma como referencia, categorías evaluativas, donde según los puntos obtenidos se le otorga una evaluación que va desde muy bien, bien, regular, mal y muy mal; lo mismo se realiza con para el promedio de carreras limpias donde según el promedio obtenido durante la competencia, recibe su evaluación con rangos iguales a los anteriormente señalados. Tabla 1

Una vez evaluados los indicadores se pasa a realizar una correlación de variables ( Pearson) para determinar el nivel o tipo de relación que se establece entre las variables puntuación obtenida en el test y el promedio de carreras limpias alcanzado en el campeonato tanto en lo individual como general, para ello se mide la categoría evaluativa alcanza- da por la puntuación y la obtenida en el promedio de carreras limpias, si los resultados en ambos indicadores son iguales se considera una relación directa, si los resultados en puntos están por encima de los resultados en cuanto a carreras limpias se considera una relación indirecta y si no se puede determinar la relación se denomina sin definir. Tabla 2

\section{Resultados}

De los 14 lanzadores analizados, ninguno es evaluado de muy bien, solo tres obtienen la categoría evaluativa de bien que representa el $21.4 \%$, cuatro lanzadores son evaluados de regular representando el $28.6 \%$ del total de la muestra y siete obtienen la categoría evaluativa de mal, lo que representa el $50 \%$ del total de los lanzadores evaluados.

En cuanto al indicador promedio de carreras limpias ningún lanzador es evaluado de muy bien, seis obtienen la evaluación de bien para el $42.8 \%$ del total de la muestra, uno de los lanzadores es evaluado de regular lo que representa el $7.14 \%$ y siete lanzadores son obtienen la evolución de muy mal lo que representa el $50 \%$ del total de la muestra tabla 2

En lo referente a la relación entre los indicadores promedio de carreras limpias y puntuación en el test se observa que ocho de los lanzadores presentan una relación directa entre los indicadores lo que representa el $57.1 \%$ del total de los sujetos evaluados, dos lanzadores presentan una relación indirecta para el 14. $3 \%$ y cuatro presentan una relación sin definir lo que representa el $28.6 \%$ del total de la muestra.

De forma general el staff de lanzadores de forma general obtiene una media en puntos de 24.67 puntos ubicándose en 
el rango o categoría evaluativa de mal y un promedio de carreras limpias de forma general de $6.18 \mathrm{pcl}$ estando evaluado con la categoría de mal.

Aunque se puede observar de forma directa la relación existente entre las variables de exposición, se realiza la prueba de correlación de Pearson para verificar si dicha relación es significativa lo cual se pudo corroborar estadísticamente al obtenerse una significación bilateral de $0.038<0.05$ tabla 3

\section{Tabla 1 Guía para evaluar los indicadores}

\begin{tabular}{llllll}
\hline Indicadores & Evaluaciones & & & \\
& Muy bien & Bien & Regular & Mal & Muy mal \\
\hline Test & $>35$ puntos & $30-34.5$ & $25-29.5$ & $20-24.5$ & $<20$ \\
Pcl & $>2.50 \mathrm{Pcl}$ & $2.51-4.0$ & $4.01-5.0$ & $5.01-6.0$ & $<6.0$ \\
\hline
\end{tabular}

Nota: $\mathrm{PCL}=$ promedio de carreras limpias por juegos.

Tabla 2 Comparación individual y general entre la puntuación del test y el promedio de carreras limpias

\begin{tabular}{|c|c|c|c|c|c|}
\hline Sujetos & Puntos & Evaluación & $\mathrm{PCL}$ & Evaluación & $\begin{array}{l}\text { Relación } \\
\text { entre } \\
\text { variables }\end{array}$ \\
\hline 1 & 23.5 & Mal & 6.36 & Muy mal & Directa \\
\hline 2 & 23.5 & Mal & 6.61 & Muy mal & Directa \\
\hline 3 & 31.0 & Bien & 3.68 & Bien & Directa \\
\hline 4 & 21.0 & Mal & 10.13 & Muy mal & Directa \\
\hline 5 & 25.0 & Regular & 9.78 & Muy mal & Sin definir \\
\hline 6 & 22.0 & Mal & 6.76 & Muy mal & Directa \\
\hline 7 & 22.0 & Mal & 12.48 & Muy mal & Directa \\
\hline 8 & 25.0 & Regular & 3.68 & Bien & Sin definir \\
\hline 9 & 33.5 & Bien & 3.17 & Bien & Directa \\
\hline 10 & 25.5 & Regular & 9.46 & Muy mal & Sin definir \\
\hline 11 & 30.5 & Bien & 3.27 & Bien & Directa \\
\hline 12 & 21.5 & Mal & 4.11 & Regular & Sin definir \\
\hline 13 & 21.5 & Mal & 3.68 & Bien & Indirecta \\
\hline 14 & 27.0 & Regular & 3.38 & Bien & Indirecta \\
\hline Totales & 24.67 & Mal & 6.18 & Mal & Directa \\
\hline
\end{tabular}


Correlaciones

Evaluación

Evaluación según

obtenida en el test $\mathrm{Pcl}$

\begin{tabular}{|c|c|c|c|}
\hline \multirow{3}{*}{$\begin{array}{l}\text { Evaluación obtenida en el } \\
\text { test }\end{array}$} & Correlación de Pearson & 1 & .559 \\
\hline & Sig.( bilateral) & & .038 \\
\hline & $\mathrm{N}$ & 14 & 14 \\
\hline \multirow[t]{3}{*}{ Evaluación según Pcl } & Correlación de Pearson & .559 & 1 \\
\hline & Sig. (bilateral) & .038 & \\
\hline & $\mathrm{N}$ & 14 & 14 \\
\hline
\end{tabular}

Nota: $\mathrm{Pcl}=$ promedio de carreras limpias, $\mathrm{N}=$ muestra

\section{Discusión}

En el estudio realizado por Cartaya (2009) realiza una valoración muy parecida a la que se hace referencia, pero se observan diferencias fundamentales sobre todo en los resultados de las puntuaciones donde los lanzadores del equipo Habana, obtienen en su mayoría evaluaciones de bien y por ende los resultados del equipo fueron muy positivos corroborando la importancia del pensamiento táctico positivo para obtener buenos dividendos en la esfera competitiva.

También se debe trabajar mucho en aspectos tácticos según situación de juego ya que se evalúan de mal de forma global a la hora de trazar estrategias y plan táctico sobre todo cuando deben lanzar con corredor en primera base y bateador derecho, dar solución al momento de enfrentar bateadores de fuerza en conteos favorables al lanzador, no saben en su mayoría en qué momento deben emplear los lanzamiento en cambios de velocidad, ni como lanzar cuando se presentan situaciones de toque de bola o posible jugada de squeze play (toque de bola con corredor en tercera menos de dos outs para que anote la carrera que casi siempre constituye la ventaja o la victoria) además de tener poca capacidad de cambiar la táctica de forma inmediata cuando el contrario no realiza lo que usted espera.

Lo anterior significa que la adquisición de la táctica atraviesa por un proceso psicopedagógico a través del cual el lanzador aprende a solucionar las situaciones que se presentan en el juego, aprovechando el potencial técnico que posee, la posibilidad de percibir una situación de juego y ofrecer una respuesta eficaz en el menor tiempo posible.

La "Dimensión táctica" de las habilidades deportivas está configurada por unos rasgos característicos tales como la captación de información relevante o la toma de decisiones en un breve lapso, y que dan sentido a la ejecución eficaz de una respuesta motriz. Al mismo tiempo, dicha dimensión táctica se pone 
se pone de relieve en la situación deportiva, en la medida en que se lleva a cabo mediante una ejecución motriz o habilidad específica, que permite ubicar en un contexto específico la decisión tomada. Reynaldo (2007) citado por Miranda (2016).

De forma general se debe hacer hincapié en la preparación táctica de los lanzadores, así como trazar estrategias que permitan obtener mejores resultados en el área de pitcheo y con ello ocupar las posiciones que ha acostumbrado a la afición de los Dorados de Chihuahua, siendo el equipo que ocupa el segundo lugar entre los que más veces han ganado el torneo estatal, detrás de los Mineros de Parral, y sobre todo por ser el equipo que representa la capital del estado, Chihuahua.

Las estadísticas generales de la competencia reflejaron sencillamente lo que se podía predecir en el test; Dorados de Chihuahua ocuparon el último lugar en promedio de efectividad (PCL) al promediar 7.93 carreras limpias por juegos, (véase en el sitio oficial de la asociación de béisbol estatal de chihuahua), muy distante del 6.06 que se obtiene como media del campeonato y más lejos aún del 4.39 de Juárez y 4.59 de Delicias que fueron los finalistas del torneo, poniendo de manifiesto que dijimos sobre la importancia de poseer un staff de pitcheo con un alto nivel de pensamiento táctico.

Otros estudios realizados hacen referencia al tema en cuestión tales como:

El pensamiento táctico y su desarrollo de López Ros, (2009), Cátedra de Deporte y Educación Física, Universidad de Girona, realiza un minucioso estudio trabajo, donde se analiza las características fundamentales del "pensamiento táctico» y hace alusión a diferentes consideraciones para su enseñanza en las etapas de iniciación y formación, fundamentalmente desde una perspectiva constructivista de corte sociocultural.

En ese sentido el autor considera que" el pensamiento táctico como un tipo de pensamiento operativo, que se muestra en la práctica, formado por un conocimiento técnico- táctico que incluye diferentes tipos de saberes, que en expertos se manifiesta como de alta calidad, y que permite guiar el comportamiento táctico en la resolución de los problemas deportivos específicos.

El estudio realizado por Mollinelo, $Y, y$ González, R (2007) acerca de variantes de ejercicios para la preparación técnico-táctica ofensiva en el equipo 15-16 años en la provincia de Villa Clara (2007) muestran una forma creativa y dinámica de cómo llevar a cabo la preparación táctica de un equipo de béisbol a través de situaciones simplificadas de juego con enfoque creativo y reproductivo.

Ejercicios para la preparación técnico-táctica en la relación lanzador receptor en el equipo de Béisbol juvenil de Villa Clara de (Hernández, V\& Hernández J, 2009). Los autores de esta investigación plantean objetivos muy parecidos a los que se proponen en la presente investigación, pero con enfoques prácticos y teóricos reflejando resultados muy importantes y útiles para su generalización. 
Conclusiones

Al analizar los resultados se pudo determinar que existe una estrecha relación entre pensamiento táctico y efectividad de los lanzadores del equipo Dorados de Chihuahua que participó en el campeonato estatal 2019 por lo que se acepta la hipótesis planteada en la investigación.

Los resultados alcanzados por el equipo en general están en correspondencia con los resultados del test, ya que el equipo ocupó el 8vo lugar del torneo y su pitcheo fue el último en efectividad y carreras permitidas.

\section{Recomendaciones}

Continuar realizándose estudios de este tipo que les permita a los entrenadores y gestores del béisbol en chihuahua, que les sirvan de herramientas para reforzar los conocimientos de esta índole y les permita trazar estrategias para mejorar tan importante aspecto y con ellos mejorar los resultados a mediano y largo plazo.

\section{Referencias}

Alvaredo, J, Hernández, O Soria, \& M Carbonell, F (1987). El pensamiento táctico en el deporte. Santa Clara. Centro Provincial de Medicina Deportiva. Resumen de trabajo de investigación. Álvarez A. Definición del Campo Táctico del Taekwondo. Revista Digital http://www.efdeportes.com - Buenos Aires - Año 8 - No 51 - agosto de 2002.

Anguera A, Blanco V, Losada J. L., \& Hernández, A. (2000): La metodología observacional en el deporte: conceptos básicos. http: //www.efdeporte. com/Revista digital -Buenos Aires año 5 - No. 24 - agosto de 2000.
Baker, D. (2001). Acute and long-term power responses to power training: Observations on the training of an elite power athlete. J Strength Cond. 23(1), 47-56.

Bompa, T., \& Buzzichelli, C. (2017). Periodización del entrenamiento deportivo (4 ${ }^{\mathrm{a}}$ ed.). Barcelona: Editorial Paidotribo.

Cañizares-Arteaga, R. G., \& Pérez-Suárez, W. (2015). Estudio biomecánico del nivel de ejecución técnica del lanzamiento a home en los lanzadores del equipo juvenil de béisbol, de Sancti Spíritus. Revista Científica Especializada en Cultura Física y Deportes, 12(26), 63-73.

Dowling, B., \& Fleisig, G. S. (2016). Kinematic comparison of baseball batting off of a tee among various competition levels. Sports biomechanics, 15(3), 255-269.

Erickson, B. J., Chalmers, P. N., Dugas, J. R., Bach Jr, B. R., Nicholson, G. P., Verma, N. N., ... \& Romeo, A. A. (2017). Do Major League Baseball Team Physicians Harvest the Semitendinosus from the Drive Leg or Landing Leg When Performing Ulnar Collateral Ligament Reconstruction on Elite Baseball Pitchers? Orthopaedic journal of sports medicine, 5(7), 2325967117713987.

Escamilla, R. F., Yamashiro, K., Mikla, T., Collins, J., Lieppman, K., \& Andrews, J. R. (2017). Effects of a short-duration stretching drill after pitching on elbow and shoulder range of motion in professional baseball pitchers. The American journal of sports medicine, 45(3), 692-700. 
Español, F. J. (2009). Evaluación Deportiva en el Béisbol: Una Batería de Tests Específicos del Béisbol. PubliCE Standard.

Fonseca, A. (2005) Béisbol preparación técnica especial. Ciudad de la Habana, Editorial Deportes. 98 p.

García, F. (1997) La psicología del deporte. Editorial de la escuela de Psicología de la Universidad San Martín, Perú.

González, G. (2001): Una aproximación practica a la psicología del deporte. http// www.efdeporte.com/Revistadigital-Buenos Aires- Año 7 - No.35 - abril 2001.

Harding, J. L., Picha, K. J., \& Bliven, K. C. H. (2018). Pitch volume andglenohumeral and hip motion and strength in youth baseball pitchers. Journal of Athletic Training, 53(1), 60-65. https://doi. org/10.4085/1062-6050-323-16 Iglesias, D. (2002) Un modelo para el análisis de los procesos cognitivos implicados en la toma de decisiones en deportes colectivos. Revista de Entrenamiento Deportivo, $16,2,9-14$.

Iglesias, D. (2003) El conocimiento y la toma de decisiones en los deportes de equipo: una revisión desde la perspectiva cognitiva. Revista de Entrenamiento Deportivo, 17, 2, 5-11.

Kolb, AY, \& Kolb, DA (2010). Aprender a jugar, jugar a aprender: un estudio de caso de un espacio de aprendizaje lúdico. Revista de Gestión del cambio organizational, 23 (1), 26-50.

La táctica individual en los deportes de equipo Mouchet, A. (2008).

López Ros, V. y Castejón, F.J. (2005) La enseñanza integrada técnico-táctica de los deportes en edad escolar. Explicación y bases de un modelo. Apuntes. Educación Física y Deportes, 79, 40-48.
Matveev. L. M. (1983). Fundamentos del entrenamiento deportivo. RDA. Editorial Científico Técnica.

Malho, F. (1974). La acción táctica en el juego. La Habana. Editorial Pueblo y Educación.

Momma, D., Funakoshi, T., Endo, K., Yokota, M., Fujisaki, K., \& Iwasaki, N. (2018). Alteration in stress distribution patterns through the elbow joint in professional and college baseball pitchers: Using computed tomography osteoabsorptiometry.

Navarro Valdivielso, F. (2015). Entrenamiento de los Jóvenes-G-SE. Revista de Entrenamiento Deportivo, 29(1).

Psicología. (1974). Libro de texto. Moscú. Editorial Fisicultura y Deportes.

Reynaldo, F. (2016). Del béisbol casi todo. Editorial Deportes.

Reynaldo, Fy Padilla, O. (2007) Tendencias actuales del entrenamiento en el Béisbol. Ciudad de La Habana, Editorial Deportes.

Roffe, M (2000): El componente psicológico en la práctica de los deportes y las actividades físicas. digital-Buenos Aires-Año 5 No.23-Julio 2000.

Siff, M. C., \& Verkhoshansky, Y. (2000). Superentrenamiento. Barcelona: Paidotribo. Verkhoshansky, Y. (1999). Todo sobre el método pliométrico. Capítulos 1 y 2. Barcelona, Editorial Paidotribo. [Fecha de acceso 23 mayo 2010].

Snyder, B., Kelmer, J., Moir, G., Davis, S., Miltenberger, M., \& Munford, S. (2016). The Relationship Between Bat Velocity and Measures of Strength and Power in Ncaa Division Ii Baseball Players: 2199 Board\# 351 June 2, 2. Medicine \& Science in Sports \& Exercise, 48(5S), 624. 


\section{Pruebas de chi-cuadrado}

\begin{tabular}{|c|c|c|c|}
\hline & Valor & df & $\begin{array}{l}\text { Significación } \\
\text { asintótica } \\
\text { (bilateral) }\end{array}$ \\
\hline Chi-cuadrado de Pearson & $99.667^{a}$ & 90 & .228 \\
\hline Razón de verosimilitud & 51.779 & 90 & 1.000 \\
\hline Asociación lineal por lineal & 2.423 & 1 & .120 \\
\hline N de casos válidos & 13 & & \\
\hline
\end{tabular}

